Article

\title{
A New Identity Involving the Chebyshev Polynomials
}

\section{Yixue Zhang and Zhuoyu Chen *}

School of Mathematics, Northwest University, Xi'an 710127, China; zhangyixue@stumail.nwu.edu.cn

* Correspondence: chenzymath@stumail.nwu.edu.cn

Received: 18 October 2018; Accepted: 5 November 2018; Published: 7 November 2018

Abstract: In this paper, firstly, we introduced a second order non-linear recursive sequence, then we use this sequence and the combinatorial methods to perform a deep study on the computational problem concerning one kind sums, which includes the Chebyshev polynomials. This makes it possible to simplify a class of complex computations involving the second type Chebyshev polynomials into a very simple problem. Finally, we give a new and interesting identity for it.

Keywords: Chebyshev polynomials; recursive sequence; convolution sums; combinatorial method; identity

MSC: Primary 11E10

\section{Introduction}

For any real number $x$, the first type Chebyshev polynomials $T_{n}(x)$ and the second type Chebyshev polynomials $U_{n}(x)$ are defined by $T_{0}(x)=1, T_{1}(x)=x, T_{2}(x)=2 x^{2}-1, T_{3}(x)=4 x^{3}-3 x$, and the second order linear recursive formula is given by:

$$
T_{n+1}(x)=2 x T_{n}(x)-T_{n-1}(x), n \geq 1 .
$$

$U_{0}(x)=1, U_{1}(x)=2 x, U_{2}(x)=4 x^{2}-1, U_{3}(x)=8 x^{3}-4 x$ and the second order linear recursive formula:

$$
U_{n+1}(x)=2 x U_{n}(x)-U_{n-1}(x), n \geq 1 .
$$

Obviously, the polynomials solution of the second order differential equation:

$$
\left(1-x^{2}\right) \frac{d^{2} y}{d x^{2}}-x \frac{d y}{d x}+n^{2} y=0,(n=0,1,2, \cdots) .
$$

are the Chebyshev polynomials in the first type $T_{n}(x)$.

The polynomials solution of the second order differential equation:

$$
\left(1-x^{2}\right) \frac{d^{2} y}{d x^{2}}-3 x \frac{d y}{d x}+n(n+2) y=0,(n=0,1,2, \cdots) .
$$

are the Chebyshev polynomials in the second type $U_{n}(x)$.

Their general expressions are:

$$
T_{n}(x)=\frac{n}{2} \sum_{k=0}^{\left[\frac{n}{2}\right]} \frac{(-1)^{k}(n-k-1) !}{k !(n-2 k) !} \cdot(2 x)^{n-2 k}, \quad(|x|<1)
$$


and:

$$
U_{n}(x)=\sum_{k=0}^{\left[\frac{n}{2}\right]} \frac{(-1)^{k}(n-k) !}{k !(n-2 k) !} \cdot(2 x)^{n-2 k},|x|<1 .
$$

The generating functions of these polynomials are:

$$
\frac{1-x t}{1-2 x t+t^{2}}=\sum_{n=0}^{\infty} T_{n}(x) t^{n}, \quad(|x|<1,|t|<1)
$$

and:

$$
\frac{1}{1-2 x t+t^{2}}=\sum_{n=0}^{\infty} U_{n}(x) t^{n},(|x|<1,|t|<1) .
$$

It rapidly becomes apparent that $T_{n}(x)$ and $U_{n}(x)$ are orthogonal polynomials. For example,

$$
\int_{-1}^{1} \sqrt{1-x^{2}} \cdot U_{m}(x) \cdot U_{n}(x) \cdot d x= \begin{cases}0 & \text { if } m \neq n \\ \frac{\pi}{2} & \text { if } m=n\end{cases}
$$

Being an essential part in both the theory and the application of mathematics, these polynomials have attracted not only the number theory experts, but also amateurs of mathematics to study their properties, which produced many interesting questions and many papers. In particular, Zhang Wenpeng [1] proved an identity including the Chebyshev polynomials and their derivative. That is:

$$
\sum_{a_{1}+a_{2}+\cdots+a_{h+1}=n} U_{a_{1}}(x) \cdot U_{a_{2}}(x) \cdots U_{a_{h}}(x) \cdot U_{a_{h+1}}(x)=\frac{1}{2^{h} \cdot h !} \cdot U_{n+h}^{(h)}(x)
$$

where the sum runs over all $(h+1)$-dimension nonnegative integer coordinates $\left(a_{1}, a_{2}, \cdots, a_{h+1}\right)$ such that $a_{1}+a_{2}+\cdots+a_{h+1}=n$, and $U_{n+h}^{(h)}(x)$ denotes the $h$-order derivative of $U_{n+h}(x)$ with respect to $x$. Then using this formula, Zhang Wenpeng [1] obtained a series of identities that consists of the Fibonacci numbers and Lucas numbers. In another direction, Ma Rong and Zhang Wenpeng [2] proved some power sums identities which were based on the nice properties of the Chebyshev polynomials. Wang Siyi [3] used the mathematical induction to prove an identity, which represent the convolution sums (2) as $U_{n}(x)$. That is:

$$
\begin{aligned}
& \sum_{a_{1}+a_{2}+\cdots+a_{k+1}=n} U_{a_{1}}(x) \cdot U_{a_{2}}(x) \cdots U_{a_{k}}(x) \cdot U_{a_{k+1}}(x) \\
= & \frac{1}{2^{k} \cdot k ! \cdot\left(1-x^{2}\right)^{k}} \cdot\left[R(n, k, x) \cdot U_{n+k-1}(x)+S(n, k, x) \cdot U_{n+k}(x)\right],
\end{aligned}
$$

where $R(n, k, x)$ and $S(n, k, x)$ are two computable polynomials of $n, k$, and $x$ with integral coefficients.

Some related theory and application results can also be found in [4-12], so they will not be repeated here.

In this paper, as a note from [1-3], we will introduce a new second order non-linear recursive sequence $C(h, j)$, then we will use this sequence to give a new and interesting expression for (2). First, we will give the main result as follows: 
Theorem 1. Let $h$ be a positive integer. If $n \geq 0$ is any integer, we obtain the identity:

$$
\begin{gathered}
\sum_{a_{1}+a_{2}+\cdots+a_{h+1}=n} U_{a_{1}}(x) U_{a_{2}}(x) \cdots U_{a_{h+1}}(x) \\
=\frac{1}{2^{h} \cdot h !} \cdot \sum_{j=1}^{h} \frac{C(h, j)}{x^{2 h-j}} \sum_{i=0}^{n} \frac{(n-i+j) !}{(n-i) !} \cdot\left(\begin{array}{c}
2 h+i-j-1 \\
i
\end{array}\right) \cdot \frac{U_{n-i+j}(x)}{x^{i}},
\end{gathered}
$$

where $C(h, i)$ is a second order non-linear recurrence sequence defined by $C(h, 0)=0, C(h, h)=1, C(h+$ $1,1)=1 \cdot 3 \cdot 5 \cdots(2 h-1)=(2 h-1) ! !$ and $C(h+1, i+1)=(2 h-1-i) \cdot C(h, i+1)+C(h, i)$ for all $1 \leq i \leq h-1$.

Compared with references [1] and [3], our Theorem 1 is very advantageous, it gives the specific form of expressing (2) as $U_{n}(x)$. Especially take $n=0$, from this theorem we now deduce the following:

Corollary 1. If any positive integer $h$ such that $h \geq 1$, we get the identity:

$$
\sum_{j=1}^{h} C(h, j) \cdot j ! \cdot x^{j} \cdot U_{j}(x)=2^{h} \cdot h ! \cdot x^{2 h}
$$

In particular, if taking $h=2$ and 3 , a slight modification of the foregoing Theorem 1 enables us to give:

Corollary 2. Given any integer $n \geq 0$, we obtain:

$$
\begin{aligned}
& \sum_{a+b+c=n} U_{a}(x) \cdot U_{b}(x) \cdot U_{c}(x)=\frac{1}{16 x^{3}} \sum_{i=0}^{n}(n+1-i)(i+2)(i+1) \cdot \frac{U_{n+1-i}(x)}{x^{i}} \\
& +\frac{1}{8 x^{2}} \sum_{i=0}^{n}(n+2-i)(n+1-i)(i+1) \cdot \frac{U_{n+2-i}(x)}{x^{i}} .
\end{aligned}
$$

Corollary 3. If $n \geq 0$ is any integer, we deduce:

$$
\begin{aligned}
& \sum_{a+b+c+d=n} U_{a}(x) \cdot U_{b}(x) \cdot U_{c}(x) \cdot U_{d}(x) \\
= & \frac{1}{384 x^{5}} \sum_{i=0}^{n}(n+1-i)(i+4)(i+3)(i+2)(i+1) \cdot \frac{U_{n+1-i}(x)}{x^{i}} \\
+ & \frac{1}{96 x^{4}} \sum_{i=0}^{n}(n+2-i)(n+1-i)(i+3)(i+2)(i+1) \cdot \frac{U_{n+2-i}(x)}{x^{i}} \\
+ & \frac{3}{96 x^{3}} \sum_{i=0}^{n}(n+3-i)(n+2-i)(n+1-i)(i+2)(i+1) \cdot \frac{U_{n+3-i}(x)}{x^{i}} .
\end{aligned}
$$

To further illustrate the application of our results, we give a numerical example. Taking $x=\frac{i}{2}$ in Corollary 2, where $i$ is an imaginary unit, i.e., $i^{2}=-1$. Note that the identity (see [1])

$$
U_{n}\left(\frac{i}{2}\right)=\frac{i^{n}}{\sqrt{5}}\left[\left(\frac{1+\sqrt{5}}{2}\right)^{n+1}-\left(\frac{1-\sqrt{5}}{2}\right)^{n+1}\right]=F_{n+1}
$$

where $F_{n}$ denotes the famous Fibonacci numbers defined by $F_{0}=0, F_{1}=1, F_{2}=1$, and $F_{n+1}=$ $F_{n}+F_{n-1}$ for all positive integers $n \geq 1$. 
From this result and Corollary 2 we may immediately deduce the following:

$$
\begin{aligned}
\sum_{a+b+c=n} F_{a+1} \cdot F_{b+1} \cdot F_{c+1}= & \frac{1}{2} \sum_{j=0}^{n}(-1)^{j} 2^{j}(n+2-j)(n+1-j)(j+1) F_{n+3-j} \\
& -\frac{1}{2} \sum_{j=0}^{n}(-1)^{j} 2^{j}(n+1-j)(j+2)(j+1) F_{n+2-j} .
\end{aligned}
$$

This shows that our results are closely related to Fibonacci numbers. Therefore, they have important application value. In other words, by our Theorem 1 we can deduce a series of important identities involving Fibonacci numbers, these fully reflect the importance of our result.

Some notes: To further understand the properties of the sequence $C(h, j)$, here we give first several values of $C(h, j)$ as follows:

$$
\begin{gathered}
C(1,1)=1 . \\
C(2,1)=1, C(2,2)=1 . \\
C(3,1)=3, C(3,2)=3, C(3,3)=1 . \\
C(4,1)=15, C(4,2)=15, C(4,3)=6, C(4,4)=1 . \\
C(5,1)=105, C(5,2)=105, C(5,3)=45, C(5,4)=10, C(5,5)=1 . \\
C(6,1)=945, C(6,2)=945, C(6,3)=420, C(6,4)=105, C(6,5)=15, C(6,6)=1 .
\end{gathered}
$$

If taking $x=1$ and noting that $U_{n}(1)=n+1$, then from Corollary 1 , we can also deduce an interesting identity involving $C(h, j)$. Therefore, we obtain:

$$
\sum_{j=1}^{h} C(h, j) \cdot(j+1) !=2^{h} \cdot h !
$$

\section{Two Simple Lemmas}

In this part, two necessary lemmas in the proof process of our theorem will be given. The first one and its detailed proof are as follows:

Lemma 1. Let $f(t)=\frac{1}{1-2 x t+t^{2}}$; if $n$ is any positive integer and $x$ and $t$ are any real numbers such that $|t|<|x|$, we have the identity:

$$
2^{n} \cdot n ! \cdot f^{n+1}(t)=\sum_{i=1}^{n} C(n, i) \cdot \frac{f^{(i)}(t)}{(x-t)^{2 n-i}}
$$

where $C(n, i)$ is the same as in Theorem 1 and $f^{(h)}(t)$ denotes the h-order derivative of $f(t)$ with respect to $t$.

Proof. Lemma 1 follows from the mathematical induction. Using the properties of the derivative, we find:

$$
f^{\prime}(t)=2(x-t) \cdot\left(1-2 x t+t^{2}\right)^{-2}=2(x-t) \cdot f^{2}(t)
$$

and:

$$
f^{\prime \prime}(t)=-2 \cdot f^{2}(t)+4(x-t) f(t) f^{\prime}(t)=-2 \cdot f^{2}(t)+8(x-t)^{2} \cdot f^{3}(t) .
$$


That is,

$$
2 \cdot f^{2}(t)=\frac{f^{\prime}(t)}{x-t}
$$

and:

$$
8 \cdot f^{3}(t)=\frac{f^{\prime \prime}(t)}{(x-t)^{2}}+\frac{f^{\prime}(t)}{(x-t)^{3}} .
$$

(3) and (4) means that Lemma 1 is true when $n=1,2$. We shall assume that Lemma 1 also holds for $2 \leq n=h$. We can obtain,

$$
2^{h} \cdot h ! \cdot f^{h+1}(t)=\sum_{i=1}^{h} C(h, i) \cdot \frac{f^{(i)}(t)}{(x-t)^{2 h-i}} .
$$

Then, from (3), (5) and the definitions of $C(k, i)$ and the derivative, we have:

$$
\begin{aligned}
& 2^{h} \cdot(h+1) ! \cdot f^{h}(t) \cdot f^{\prime}(t)=2^{h+1} \cdot(h+1) !(x-t) \cdot f^{h+2}(t) \\
= & \sum_{i=1}^{h} \frac{(2 h-i) \cdot C(h, i)}{(x-t)^{2 h+1-i}} \cdot f^{(i)}(t)+\sum_{i=1}^{h} \frac{C(h, i)}{(x-t)^{2 h-i}} \cdot f^{(i+1)}(t) \\
= & \frac{(2 h-1) C(h, 1)}{(x-t)^{2 h}} \cdot f^{\prime}(t)+\sum_{i=1}^{h-1} \frac{(2 h-1-i) C(h, i+1)}{(x-t)^{2 h-i}} \cdot f^{(i+1)}(t) \\
& +\frac{C(h, h)}{(x-t)^{h}} \cdot f^{(h+1)}(t)+\sum_{i=1}^{h-1} \frac{C(h, i)}{(x-t)^{2 h-i}} \cdot f^{(i+1)}(t) \\
= & \frac{(2 h-1) C(h, 1)}{(x-t)^{2 h}} \cdot f^{\prime}(t)+\frac{C(h, h)}{(x-t)^{h}} \cdot f^{(h+1)}(t) \\
& +\sum_{i=1}^{h-1} \frac{(2 h-1-i) C(h, i+1)}{(x-t)^{2 h-i} \cdot f^{(i+1)}(t)+\sum_{i=1}^{h-1} \frac{C(h, i)}{(x-t)^{2 h-i}} \cdot f^{(i+1)}(t)} \\
= & \frac{C(h+1,1)}{(x-t)^{2 h} \cdot f^{\prime}(t)+\frac{C(h+1, h+1)}{(x-t)^{h}} \cdot f^{(h+1)}(t)} \\
& +\sum_{i=1}^{h-1} \frac{C(h+1, i+1)}{(x-t)^{2 h-i}} \cdot f^{(i+1)}(t) \\
= & \frac{C(h+1,1)}{(x-t)^{2 h} \cdot f^{\prime}(t)+\frac{C(h+1, h+1)}{(x-t)^{h}} \cdot f^{(h+1)}(t)+\sum_{i=2}^{h} \frac{C(h+1, i)}{(x-t)^{2 h+1-i}} \cdot f^{(i)}(t)} \\
= & \sum_{i=1}^{h+1} C(h+1, i) \cdot \frac{f^{(i)}(t)}{(x-t)^{2 h+1-i}} \cdot
\end{aligned}
$$

From (6), we may immediately deduce that:

$$
2^{h+1} \cdot(h+1) ! \cdot f^{h+2}(t)=\sum_{i=1}^{h+1} C(h+1, i) \cdot \frac{f^{(i)}(t)}{(x-t)^{2 h+2-i}} .
$$

That is, Lemma 1 also holds if $n=h+1$. Now, the conclusion of Lemma 1 follows from the mathematical induction.

If we let $g(t)=\frac{1-x t}{1-2 x t+t^{2}}$. Then we are not easy to deduce a formula similar to Lemma 1 . That is why we have not give a corresponding result of the first type Chebyshev polynomials in the theorem. 
Lemma 2. For any positive integers $h$ and $k$, the power series expansion is given by the formula:

$$
\frac{f^{(h)}(t)}{(x-t)^{k}}=\frac{1}{x^{k}} \sum_{n=0}^{\infty}\left(\sum_{i=0}^{n} \frac{(n-i+h) !}{(n-i) !} \cdot U_{n-i+h}(x) \cdot\left(\begin{array}{c}
i+k-1 \\
i
\end{array}\right) \cdot \frac{1}{x^{i}}\right) t^{n}
$$

for all real numbers $t$ and $x$ with $|t|<|x|$.

Proof. Actually, this result is easily deduced by the properties of the power series. Firstly, the formula:

$$
f(t)=\frac{1}{1-2 x t+t^{2}}=\sum_{n=0}^{\infty} U_{n}(x) \cdot t^{n}
$$

can be derived from the definition of the second type Chebyshev polynomials $U_{n}(x)$. Then, let $h$ be any positive integer; applying the properties of the power series, we acquire:

$$
\begin{aligned}
& f^{(h)}(t)=\sum_{n=0}^{\infty}(n+h)(n+h-1) \cdots(n+1) \cdot U_{n+h}(x) \cdot t^{n} \\
= & \sum_{n=0}^{\infty} \frac{(n+h) !}{n !} \cdot U_{n+h}(x) \cdot t^{n} .
\end{aligned}
$$

Second, if $k$ is a positive integer. Then, for all real $t$ and $x$, which satisfied $|t|<|x|$, the power series expansion is given by:

$$
\frac{1}{x-t}=\frac{1}{x} \cdot \sum_{n=0}^{\infty} \frac{t^{n}}{x^{n}}
$$

Similarly,

$$
\begin{aligned}
\frac{1}{(x-t)^{k}} & =\frac{1}{(k-1) ! \cdot x^{k}} \cdot \sum_{n=0}^{\infty}(n+k-1)(n+k-2) \cdots(n+1) \cdot \frac{t^{n}}{x^{n}} \\
& =\frac{1}{x^{k}} \cdot \sum_{n=0}^{\infty}\left(\begin{array}{c}
n+k-1 \\
n
\end{array}\right) \cdot \frac{t^{n}}{x^{n}} .
\end{aligned}
$$

Combining (7), (8) and concerned with the multiplicative properties of the power series, we obtain:

$$
\begin{aligned}
& \frac{f^{(h)}(t)}{(x-t)^{k}}=\frac{1}{x^{k}} \cdot\left(\sum_{n=0}^{\infty} \frac{(n+h) !}{n !} \cdot U_{n+h}(x) \cdot t^{n}\right)\left(\sum_{n=0}^{\infty}\left(\begin{array}{c}
n+k-1 \\
n
\end{array}\right) \cdot \frac{t^{n}}{x^{n}}\right) \\
= & \frac{1}{x^{k}} \sum_{n=0}^{\infty}\left(\sum_{i+j=n} \frac{(j+h) !}{j !} \cdot U_{j+h}(x) \cdot\left(\begin{array}{c}
i+k-1 \\
i
\end{array}\right) \cdot \frac{1}{x^{i}}\right) t^{n} \\
= & \frac{1}{x^{k}} \sum_{n=0}^{\infty}\left(\sum_{i=0}^{n} \frac{(n-i+h) !}{(n-i) !} \cdot U_{n-i+h}(x) \cdot\left(\begin{array}{c}
i+k-1 \\
i
\end{array}\right) \cdot \frac{1}{x^{i}}\right) t^{n} .
\end{aligned}
$$

That is, Lemma 2 has been proven.

\section{Proof of the Theorem}

In Section 2, we proved two lemmas that play a significant role in the proof process of the theorem. We turn now to a detailed exhibit of Theorem 1. In fact, if $h$ is a positive integer, combined with the properties of the power series, we use (1), Lemma 1 and the definition of $f(t)$ to get:

$$
2^{h} \cdot h ! \cdot f^{h+1}(t)=2^{h} \cdot h ! \cdot\left(\sum_{n=0}^{\infty} U_{n}(x) \cdot t^{n}\right)^{h+1}
$$




$$
=2^{h} \cdot h ! \cdot \sum_{n=0}^{\infty}\left(\sum_{a_{1}+a_{2}+\cdots+a_{h+1}=n} U_{a_{1}}(x) U_{a_{2}}(x) \cdots U_{a_{h+1}}(x)\right) \cdot t^{n} .
$$

In another way, applying Lemma 2, we can also obtain:

$$
\begin{aligned}
& 2^{h} \cdot h ! \cdot f^{h+1}(t)=\sum_{j=1}^{h} C(h, j) \cdot \frac{f^{(j)}(t)}{(x-t)^{2 h-j}} \\
= & \sum_{j=1}^{h} \frac{C(h, j)}{x^{2 h-j}} \cdot\left(\sum_{n=0}^{\infty}\left(\sum_{i=0}^{n} \frac{(n-i+j) !}{(n-i) !} \cdot U_{n-i+j}(x) \cdot\left(\begin{array}{c}
2 h+i-j-1 \\
i
\end{array}\right) \cdot \frac{1}{x^{i}}\right) t^{n}\right) \\
= & \sum_{n=0}^{\infty}\left(\sum_{j=1}^{h} \frac{C(h, j)}{x^{2 h-j}} \sum_{i=0}^{n} \frac{(n-i+j) !}{(n-i) !} \cdot \frac{U_{n-i+j}(x)}{x^{i}} \cdot\left(\begin{array}{c}
2 h+i-j-1 \\
i
\end{array}\right)\right) \cdot t^{n} .
\end{aligned}
$$

Combining (9), (10) and Lemma 1, as well as comparing the coefficients of the power series, we can instantly deduce the identity:

$$
\begin{aligned}
& 2^{h} \cdot h ! \sum_{a_{1}+a_{2}+\cdots+a_{h+1}=n} U_{a_{1}}(x) U_{a_{2}}(x) \cdots U_{a_{h+1}}(x) \\
= & \sum_{j=1}^{h} \frac{C(h, j)}{x^{2 h-j}} \sum_{i=0}^{n} \frac{(n-i+j) !}{(n-i) !} \cdot\left(\begin{array}{c}
2 h+i-j-1 \\
i
\end{array}\right) \cdot \frac{U_{n-i+j}(x)}{x^{i}} .
\end{aligned}
$$

In summary, we have completed the proof of Theorem 1.

\section{Conclusions}

The main result of this paper is Theorem 1. It gives a new representation of the summation (2). The feature of this theorem is simple and clear, and its right side is represented as a linear combination of $U_{i}(x)$, which is very easy to calculate. Furthermore, our research methods can also be applied to many other special polynomials; for instance, Bell polynomials, Fibonacci polynomials, Lucas polynomials, etc.

Corollary 1 profoundly reveals the close relationship between the second type Chebyshev polynomials themselves. That is to say, it characterizes the relationship between the standard orthogonal basis $\left\{1, x, x^{2}, x^{3}, \ldots, x^{h}\right\}$ and the orthogonal basis $\left\{U_{0}(x), U_{1}(x), U_{3}(x), \cdots, U_{h}(x)\right\}$.

For the first type Chebyshev polynomials $T_{n}(x)$, we naturally ask whether there exists a similar formula as in our Theorem 1. This still is an open problem. It will be studied in the future.

Author Contributions: Writing, original draft: Y.Z. Writing, review and editing: Z.C.

Funding: This research was funded by NSF (11771351) of China.

Acknowledgments: The authors would like to thank the referees for their very helpful and detailed comments, which have significantly improved the presentation of this paper.

Conflicts of Interest: The authors declare no conflict of interest.

\section{References}

1. Zhang, W. Some identities involving the Fibonacci numbers and Lucas numbers. Fibonacci Q. 2004, 42, 149-154.

2. Ma, R.; Zhang, W. Several identities involving the Fibonacci numbers and Lucas numbers. Fibonacci Q. 2007, $45,164-170$.

3. Wang, S.Y. Some new identities of Chebyshev polynomials and their applications. Adv. Differ. Equ. 2015, $2015,335$.

4. Chen, L.; Zhang, W. Chebyshev polynomials and their some interesting applications. Adv. Differ. Equ. 2017, 2017, 303. 
5. Li, X.X. Some identities involving Chebyshev polynomials. Math. Probl. Eng. 2015, 2015, 950695. [CrossRef]

6. Ma, Y.; Lv, X.-X. Several identities involving the reciprocal sums of Chebyshev polynomials. Math. Probl. Eng. 2017, 2017, 4194579. [CrossRef]

7. Cesarano, C. Identities and generating functions on Chebyshev polynomials. Georgian Math. J. 2012, 19, 427-440. [CrossRef]

8. Cesarano, C. Integral representations and new generating functions of Chebyshev polynomials. Hacettepe J. Math. Stat. 2015, 44, 535-546. [CrossRef]

9. Bircan, N.; Pommerenke, C. On Chebyshev polynomials and GL(2,Z/pZ). Bull. Math. Soc. Sci. Math. Roum. 2012, 103, 353-364.

10. Lee, C.-L.; Wong, K.-B. On Chebyshev's Polynomials and Certain Combinatorial Identities. Bull. Malays. Math. Sci. Soc. 2011, 34, 279-286.

11. Wang, T.; Zhang, H. Some identities involving the derivative of the first kind Chebyshev polynomials. Math. Probl. Eng. 2015, 2015, 146313. [CrossRef]

12. Wang, T.; Zhang, W. Two identities involving the integral of the first kind Chebyshev polynomials. Bull. Math. Soc. Sci. Math. Roum. 2017, 108, 91-98.

(C) 2018 by the authors. Licensee MDPI, Basel, Switzerland. This article is an open access article distributed under the terms and conditions of the Creative Commons Attribution (CC BY) license (http://creativecommons.org/licenses/by/4.0/). 